http://dx.doi.org/10.21611/qirt.1992.031

\title{
A quantitative analysis of pulsed video thermography
}

\author{
D. P. ALMOND and S. K. LAU*
}

School of Materials Science, University of Bath, Bath, BA2 TAY, UK.

\begin{abstract}
The defect imaging capability of a pulsed video thermography system is assessed by a quantitative analysis of its performance in imaging well characterised artificial defects. The experimental data show marked deviations from the predictions of one-dimensional heat flow theory. An analytical model is presented which provides a basic understandings of the imaging process for finite sized defects. A method for the determination of defect depth is. proposed.
\end{abstract}

\section{Introduction}

Pulsed video thermography (PVT) was developed by Milne and Reynolds [1] specifically for non-destructive evaluation (NDE). A PVT system comprises an infrared video camera and a high intensity flash tube which is used to flash heat the surface of the part under inspection. The resulting thermal transient can be recorded on synchronised video tape or within the computer control system of the camera. The latter is particularly convenient and has great potential for image processing and image analysis.

The PVT technique provides a rapid means of obtaining images of defects which are caused by the effects they have on heat flow into the test-piece away from the heated surface. The process of image formation is closely related to that accounting for thermal wave images of sub-surface features. It is essential with all NDE techniques to develop a quantitative understanding of the relationships between measureable defect responses and the size, shape, orientation and location of the defect concerned. This work was designed to address this problem by obtaining quantitative data of the PVT response of well characterised defects followed by the development of an appropriate model of thermal image formation.

\section{Experimental}

The test-piece that was studied is shown schematically in figure 1 . It was a mild steel block which contained a set of circular air gaps ranging in size from 0.5 to $10 \mathrm{~mm}$ diameter at a depth of about $0.7 \mathrm{~mm}$ beneath the surface. The surface of the block was blackened to ensure uniform emissivity. The artificial defect diameters were chosen to obtain an indication of the resolution of the technique and to facilitate a study of the effect of defect size on thermal response.

The PVT images of the test-piece were obtained at the National NDT Centre, Harwell, UK employing a system which has been described before [1]. Clear images were obtained of all the defects with diameters greater than $1 \mathrm{~mm}$. A quantitative analysis of the video recording was carried out at the University of Bath using an image analyser.

The PVT images of the defects in the test-piece were caused by the steel above the air gaps becoming hotter on flash heating and cooling slower than the adjacent metal. The images of the defects persisted for many frames after the flash, although the images of the smaller defects faded long before that of the largest. The image analyser was used to obtain the thermal profiles across the images and to determine how these altered with time. An example of a set of thermal profiles taken from the images of the $4 \mathrm{~mm}$ diameter defect is shown in figure 2. The defect diameter is indicated in the figure and it is clear that the images have a similar size. It is not clear how to determine the precise size of the defect from these images. 
Throughout this work the thermal contrast is shown which is the difference between the temperature of the defect image and that of the surrounding non-defective areas of the testpiece. The temporal behaviour of the images are indicated in figure 3 which shows the time dependences of the thermal contrast at the centres of the defect images. The temporal behaviour is clearly highly dependent on defect dimension. The peak in defect contrast not only increases in amplitude with defect size but it also occurs at later times. Since these defects were all at the same depth beneath the surface, this is quite at variance with the predictions of one-dimensional thermal conduction theory and indicates the necessity of the development of a more appropriate model which takes account of the finite size of real defects.

\section{Finite Defect Response Analysis.}

As a first order approximation, the defects are treated as being perfectly reflecting disks of radius a set at a depth $z$ below the surface. It is assumed that the surface is heated by a pulse of energy which may be approximated by a Dirac delta function at time $t=0$. The subsequent thermal transient may be obtained by first solving the problem in the frequency domain and then performing a Laplace inversion. Employing this strategy here amounts to first calculating the image on the surface caused by a periodically heated disk. This image may be obtained employing a Huygens' construction [2] in which each point on the surface of the disk becomes a secondary source emitting a spherical thermal wave front with wave vector $\sigma=(1+i) / \mu$ where $\mu$ is the thermal diffusion length. Following a Laplace inversion, the required solution for a pulsed heated surface is:

$$
T(x, t)=\frac{2 I_{0}}{\pi k} \int_{0}^{a} \int_{0}^{\pi} \frac{\exp \left[-(z+R)^{2} / 4 \alpha t\right]}{2 \sqrt{\pi \alpha t^{3}}} \times \frac{(z+R)}{R} r d \theta d r
$$

in which $T(x, t)$ is the temperature on the surface at a distance $x$ from the centre of the defect image at a time $t$ after the pulse of intensity $10 ; R$ is the distance of a source element of area $r d \theta d r$ on the disk from the point $x$ and $k$ and a are the thermal conductivity and diffusivity. Numerical computations of this expression corresponding with the $4 \mathrm{~mm}$ diameter defect are shown in figure 4. These are found to be in good qualitative agreement with the experimental data, figure 2. However, the experimental images are somewhat narrower than the computations and they decay more rapidly. The computed time dependences for the centres of the defect images are shown in figure 5. Again these have the same general characteristics as the experimental data in figure 3 although the contrast-time peaks are much narrower than observed for the larger diameter defects. It is notable that these peaks increase in magnitude and occur at later times as defect diameter is increased, in agreement with the experimental data and in sharp contrast with the predictions of one-dimensional heat flow theory.

This first order model successfully reproduces the main characteristics of PVT images. An improvement, which has already been reported [3], is to add thermal reverberations between the defect and the surface. These significantly broaden the contrast-time peaks of the larger defects and seem to be an essential part of the long time response. A further improvement which will be the subject of a future publication is to apply a realistic temperature distribution to the periodically heated disk. Exact calculations show the temperature profile to fall to zero, over a distance $\mu$, at the edge of a crack rather than being uniform up to the edge of the defect as assumed here. The effect of this is to reduce the widths of the predicted thermal images, accounting for some of the differences between figures 2 and 4 .

\section{Defect Depth}

This work has shown that the time of peak image contrast is not a good indicator of defect depth because it depends of defect diameter. However, it is evident in both figures 3 and 5 that the initial slope of the contrast-time peaks is shared by the defects of all sizes. It can be 


\section{http://dx.doi.org/10.21611/qirt.1992.031}

shown analytically [3] that this slope varies with defect depth and it is independent of defect size. Consequentiy, it is suggested that the short-time slope of the contrast-time curve, rather than the peak time, is used to assess defect depth.

\section{Conclusions}

The simple first-order model reported here seems to provide a basic framework for understanding the PVT imaging process. Experimentally it is found that the most comprehensive images are those obtained at short-times ie in early frames soon after the excitation flash. This is fortunate as at these short- times the predictions of the basic model are close to the data. The long-time decay of the images is not accurately accounted for by the model, but it may be argued that this is less important because long-time data is of less intrinsic value.

\section{Acknowledgements}

One of the authors, S K Lau, is grateful to PowerGen for the studentship that supported his work on this project.

\section{REFERENCES}

[1] MILNE (J.M.) AND REYNOLDS (W.N).-Application of thermal pulses and infrared thermal images for observing subsurface structures in metals and composites. SPIE, 590, 1985, p.293302.

[2] GREEN (G).-On a fundamental problem in diffraction. Phil.Mag. 21, 1936, p.934-947.

[3] LAU (S.K.), ALMOND (D.P.) and MILNE (J.M.).-A quantitative analysis of pulsed video thermography. NDT\&E Int. 24, 1991, p.195-202.

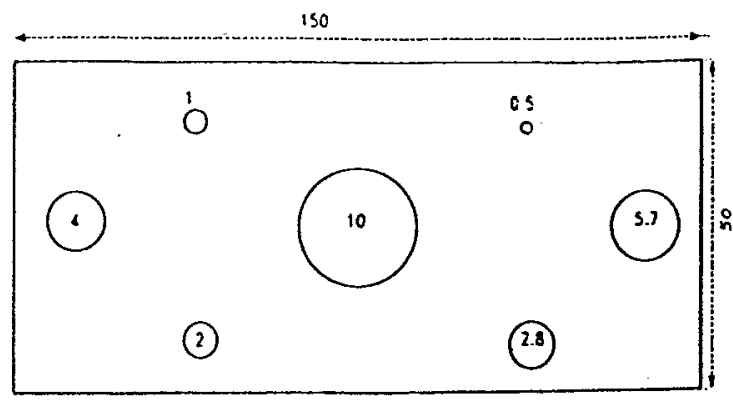

TOP VIEH

All Dimensions in $\mathrm{mm}$

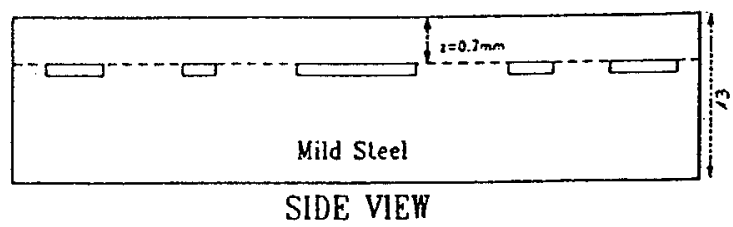

Fig. 1. - Schematic diagram of the test-plece, (a) plan; (b) section. All dimensions in $\mathrm{mm}$. 
http://dx.doi.org/10.21611/qirt.1992.031

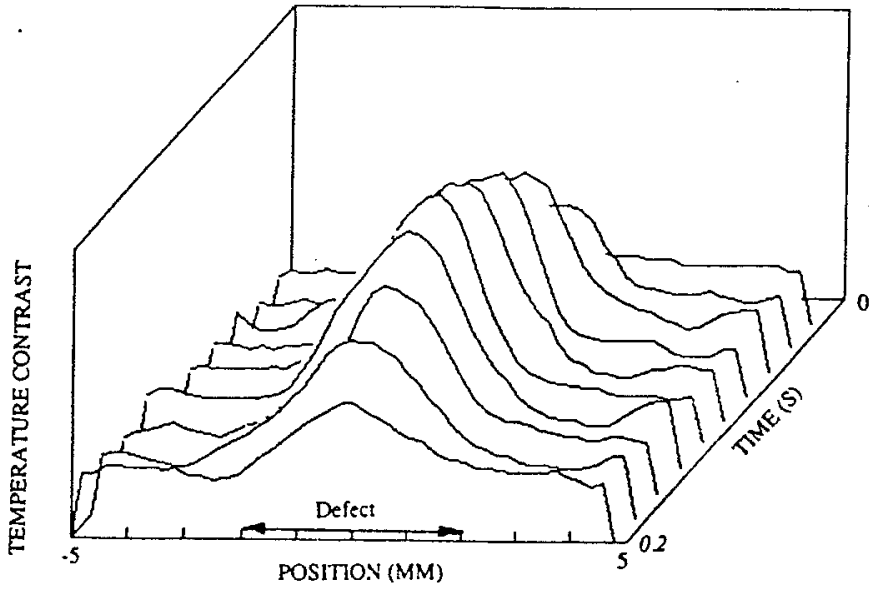

Fig. 2. - Temperature contrast profiles across the centres of successive PVT Images of the $4 \mathrm{~mm}$ dlameter defect.

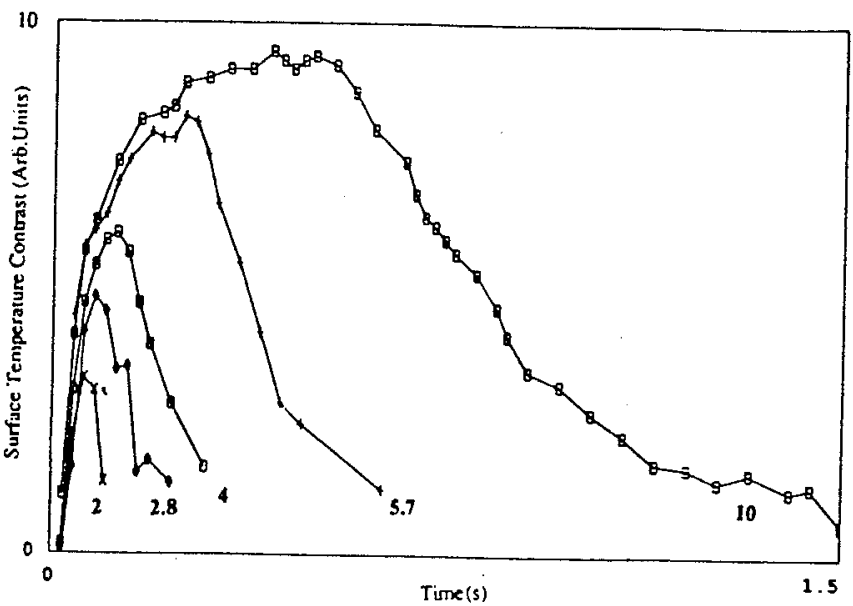

Fig. 3. - TIme dependences of the thermal contrast of the PVT images of the 2, 2.8, 4, 5.7 and 10 $\mathrm{mm}$ diameter defects. 
http://dx.doi.org/10.21611/qirt.1992.031

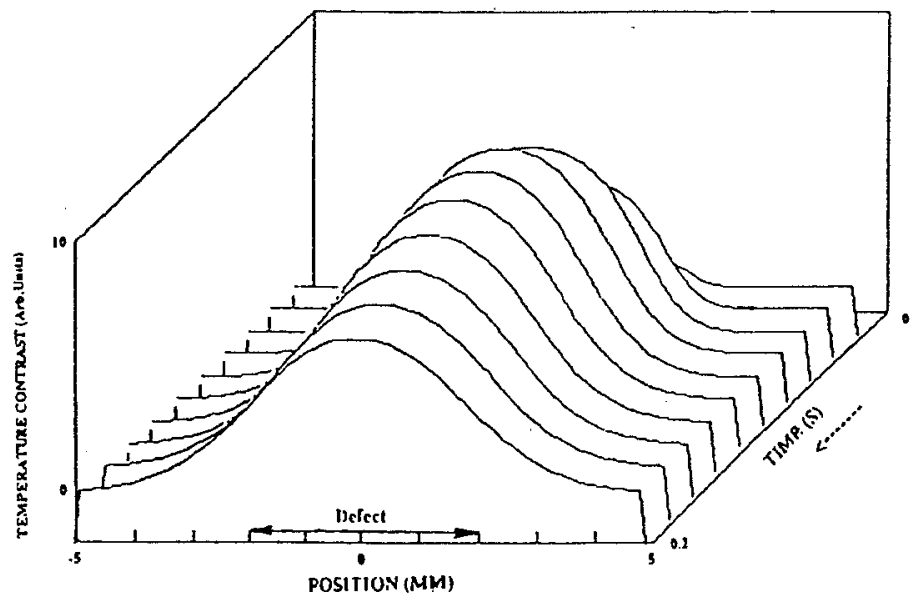

Fig. 4. - Predicted thermal contrast line profiles across the centres of Images of the $4 \mathbf{m m}$ diameter defect.

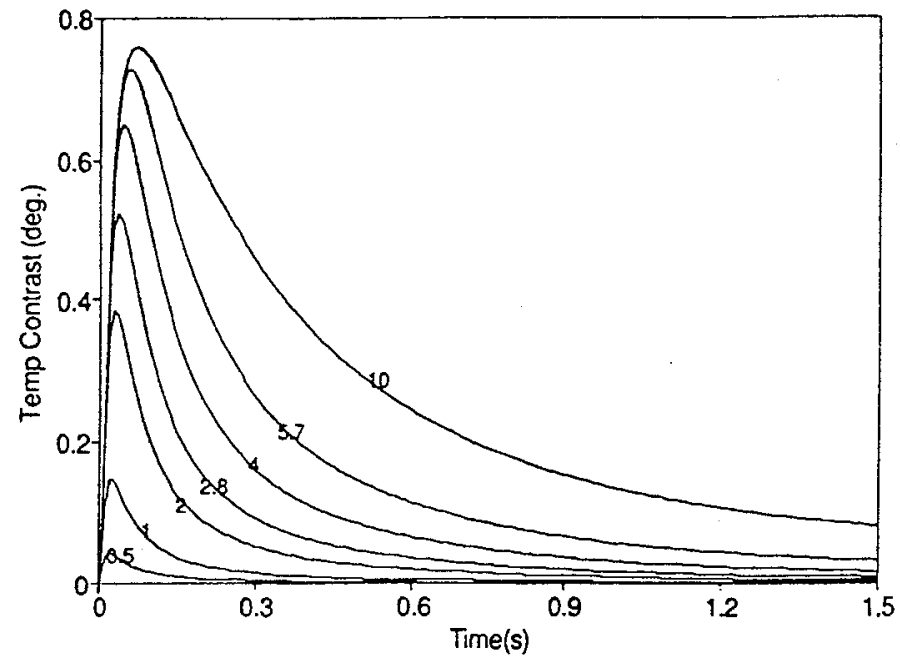

Fig. 5. - Predicted time dependences of the thermal contrasts of the test-piece defect images. 